\title{
Lambda-Statistical Limit Inferior and Limit Superior for Sequences of Fuzzy Numbers
}

\author{
F. Berna Benli
}

\begin{abstract}
Aytar, Mammadov and Pehlivan have introduced the concepts of statistical limit inferior and limit superior for sequences of fuzzy numbers. In this paper, we define lamda-statistical limit inferior and limit superior for sequences of fuzzy numbers. Also we will discuss the relations among, lambda-statistical limit inferior and limit superior for sequences of fuzzy numbers
\end{abstract}

Index Terms-Fuzzy number, lambda-statistical limit inferior, lambda-statistical limit superior.

\section{INTRODUCTION}

The idea of statistical convergence of a sequence was introduced by Steinhaus [1] and Fast [2]. Statistical convergence was generalized by Buck [3].

Matloka [4] was defined bounded and convergent sequences of fuzzy numbers, also showed that every convergent sequence is bounded. Later, Nanda [5] studied the spaces of bounded and convergent sequences of fuzzy numbers and shoved that these are complete metric spaces. Then, Nuray and Savas [6] have extended and also discussed the concepts of statistically convergent and statistically Cauchy sequences of fuzzy numbers. Lambda-statistically Cauchy sequences of fuzzy numbers have been introduced by Tuncer and Benli [7]. Also, Tuncer and Benli [8] have defined lambda-statistical limit and lambda-statistical cluster points of a sequence of fuzzy numbers and the concepts of lambda-statistically monotonic and lambda-statistically bounded sequences of fuzzy numbers have been given in [9].

Recently, sup and inf notions have been given only for bounded sets of fuzzy numbers ( [10] and [11] ). Then in [12] Aytar, Mamedov and Pehlivan have introduced the concepts of statistical limit inferior and limit superior for statistically bounded sequences of fuzzy numbers.

In this paper we will define lamda-statistical limit inferior and limit superior for sequences of fuzzy numbers, then we will prove that some results established for sequences of real numbers [13] are also valid for sequences of fuzzy numbers.

Shortly, we recall some of the basic notations in the theory of fuzzy numbers.

Let $D$ denote the set of all closed bounded intervals $A=[\underline{A}, \bar{A}]$ on the real line $R$. For $A, B \in D$ define

$$
A \leq B \Leftrightarrow \underline{A} \leq \underline{B} \text { and } \bar{A} \leq \bar{B},
$$

Manuscript received April 20, 2013; revised June 19, 2013. This work is supported by the Scientific Research Center at the Erciyes University with the project code FBA-12-4137.

F. Berna Benli is with Department of Mathematics, Erciyes University, Turkey(e-mail: akpinarb@erciyes.edu.tr).

$$
d(A, B)=\max (|\underline{A}-\underline{B}|,|\bar{A}-\bar{B}|)
$$

It is easy to see that $d$ defines a metric on $D$ and $(d, D)$ is a complete metric space. Also $\leq$ is a partial order in $D$.

A fuzzy number is a fuzzy subset of real line $R$ which is bounded, convex and normal. Let $L(R)$ denote the set of all fuzzy numbers which are upper semicontinuous and have compact support. In other words, if $X \in L(R)$, then for any $\alpha \in[0,1], X^{\alpha}$ is compact, where

$$
X^{\alpha}= \begin{cases}t: X(t) \geq \alpha & \text { if } \alpha \in(0,1] \\ t: X(t)>0 & \text { if } \alpha=0\end{cases}
$$

Define a map $\bar{d}: L(R) \times L(R) \rightarrow R$ by

$$
\bar{d}(X, Y)=\sup _{\alpha \in[0,1]} d\left(X^{\alpha}, Y^{\alpha}\right) .
$$

It is known that ( [14] ) $L(R)$ is a complete metric space with the metric $\bar{d}$.

For $X, Y \in L(R)$ define $X \leq Y$ if and only if $X^{\alpha} \leq Y^{\alpha}$ for any $\alpha \in[0,1]$.

The fuzzy numbers $X$ and $Y$ are said to be incomparable if neither $X \leq Y$ nor $Y \leq X$. We use the notation $X \psi_{Y}$ in this case.

For every $X, Y, Z \in L(R)$, we say that $Z$ is the sum of $X$ and $Y$, written $Z=X+Y$, if for every $\alpha \in[0,1]$, $\underline{Z}^{\alpha}=\underline{X}^{\alpha}+\underline{Y}^{\alpha}$ and $\bar{Z}^{\alpha}=\bar{X}^{\alpha}+\bar{Y}^{\alpha}$.

Consider a fuzzy number $\mu \in L(R)$. Let $\mu^{\alpha}=\left[\mu^{\alpha}, \bar{\mu}^{\alpha}\right], \alpha \in[0,1]$, be $\alpha$ - level sets of $\mu$. Given a positive number $a>0$, we define the fuzzy numbers $\mu+a_{1}$ and $\mu-a_{1}$ as follows [15]

$$
\begin{aligned}
& \left(\mu+a_{1}\right)^{\alpha}=\left[\underline{\mu}^{\alpha}, \bar{\mu}^{\alpha}\right]+[a, a]=\left[\underline{\mu}^{\alpha}+a, \bar{\mu}^{\alpha}+a\right], \\
& \left(\mu-a_{1}\right)^{\alpha}=\left[\underline{\mu}^{\alpha}, \bar{\mu}^{\alpha}\right]-[a, a]=\left[\underline{\mu}^{\alpha}-a, \bar{\mu}^{\alpha}-a\right],
\end{aligned}
$$

where

$$
a_{1}(x)= \begin{cases}1, & x=a \\ 0, & \text { otherwise }\end{cases}
$$

Clearly $\mu+a_{1}, \mu-a_{1} \in L(R)$. In addition, we have $\mu-a_{1}<\mu<\mu+a_{1}$ and $\bar{d}\left(\mu, \mu+a_{1}\right)=\bar{d}\left(\mu, \mu-a_{1}\right)=a$. 
A sequence $X=\left(X_{k}\right)$ of fuzzy numbers is said to be bounded if the set $\left\{X_{k}: k \in N\right\}$ of fuzzy numbers is bounded.

Definition 1.1. The sequence $X=\left(X_{k}\right)$ is said to be convergent to the fuzzy number $\left(X_{0}\right)$, written as $\lim _{k} X_{k}=X_{0}[6]$, if for every $\varepsilon>0$ there exists a positive integer $n=n_{0}(\varepsilon)$ such that

$$
\bar{d}\left(X_{k}, X_{0}\right)<\varepsilon \text { for every } k>n_{0} .
$$

If $K$ is a subset of positive integers $N$, then $K_{n}$ denotes the set $\{k \in K: k \leq n\}$. The natural density of $K$ is given by $\delta(K)=\lim _{n \rightarrow \infty} \frac{\left|K_{n}\right|}{n}$, where $\left|K_{n}\right|$ denotes the number of elements in $K_{n}$. Clearly, finite subsets have zero natural density and $\delta\left(K^{c}\right)=1-\delta(K)$ where, $K^{c}=N-K$ is the complement of $K$. If $K_{1} \subseteq K_{2}$, Then $\delta\left(K_{1}\right) \leq \delta\left(K_{2}\right)$ (see [16] ). For a sequence of real numbers $x=\left(x_{k}\right)$, the notions of statistical limit superior and limit inferior are defined as follows [13];

$$
\begin{aligned}
& s t-\lim \sup x=\left\{\begin{array}{l}
\sup B_{x}, \quad B_{x} \neq \phi \\
-\infty, \text { otherwise }
\end{array}\right. \\
& \text { st }-\lim \inf x=\left\{\begin{array}{l}
\inf A_{x}, \quad A_{x} \neq \phi \\
+\infty, \text { otherwise }
\end{array}\right.
\end{aligned}
$$

where $A_{x}=\left\{a \in R: \delta\left(\left\{k \in N: x_{k}<a\right\}\right) \neq 0\right\}$ and

$$
B_{x}=\left\{b \in R: \delta\left(\left\{k \in N: x_{k}>b\right\}\right) \neq 0\right\} .
$$

Definition 1.2. The sequence of fuzzy numbers $X=\left(X_{k}\right)$ is statistically convergent to $X_{0}$ if the set $\left\{k \in N: \bar{d}\left(X_{k}, X_{0}\right) \geq \varepsilon\right\}$ has natural density zero for every $\varepsilon>0$. We will use the notation $s t-\lim X_{k}=X_{0}$ as in [6].

The sequence $X=\left(X_{k}\right)$ is said to be statistically bounded from above if there exists a fuzzy number $\mu$ ( called the statistical upper bound) such that

$$
\delta\left(\left\{k \in N: X_{k}>\mu\right\} \cup\left\{k \in N: X_{k} \nsucc \mu\right\}\right)=0
$$

The statistical lower bound can be defined similarly.

If the sequence $X=\left(X_{k}\right)$ is both statistically bounded from above and statistically bounded from below then it is called statistically bounded [17].

Definition 1.3. ( [18] ) Let $I_{n}=\left[n-\lambda_{n}+1, n\right], \lambda=\left(\lambda_{n}\right)$ be a non-decreasing sequence of positive numbers tending to $\infty, \lambda_{n+1} \leq \lambda_{n}+1, \lambda_{1}=1$ and $X=\left(X_{k}\right)$ be a sequence of fuzzy numbers. A sequence $X=\left(X_{k}\right)$ of fuzzy number is said to be $\lambda$-statistically convergent or $s \lambda$-convergent to fuzzy numbers $X_{0}$, written as $s \lambda-\lim X_{k}=X_{0}$ if for every $\varepsilon>0$

$$
\begin{gathered}
\lim _{n} \frac{1}{\lambda_{n}}\left|\left\{k \in I_{n}: \bar{d}\left(X_{k}, X_{0}\right) \geq \varepsilon\right\}\right|=0 \text { or } \\
\delta_{\lambda}\left(\left\{k \in I_{n}: \bar{d}\left(X_{k}, X_{0}\right) \geq \varepsilon\right\}\right)=0
\end{gathered}
$$

The sequence $X=\left(X_{k}\right)$ is said to be $\lambda$-statistically bounded from above if there exists a fuzzy number $M$ called the $\lambda$-statistical upper bound) such that

$$
\delta_{\lambda}\left(\left\{k \in I_{n}: X_{k}>M\right\} \cup\left\{k \in I_{n}: X_{k} \nsucc M\right\}\right)=0
$$

The $\lambda$-statistical lower bound can be defined similarly.

If the sequence $X=\left(X_{k}\right)$ is both $\lambda$-statistically bounded from above and $\lambda$-statistically bounded from below then it is called $\lambda$-statistically bounded [9].

\section{LAMBDA STATISTICAL LIMIT INFERIOR AND LIMIT SUPERIOR FOR SEQUENCES OF FUZZY NUMBERS}

In this section, we introduce the concepts of $\lambda$-statistically limit superior and limit inferior for $\lambda$-statistically bounded sequences of fuzzy numbers. Given a sequence $X=\left(X_{k}\right)$ let us define:

$$
\begin{aligned}
& A_{X}^{\lambda}=\left\{M \in L(R): \lim _{n} \frac{1}{\lambda_{n}}\left|\left\{k \in I_{n}: X_{k}<M\right\}\right| \neq 0\right\} \\
& \overline{A_{X}^{\lambda}}=\left\{M \in L(R): \lim _{n} \frac{1}{\lambda_{n}}\left|\left\{k \in I_{n}: X_{k}>M\right\}\right|=1\right\} \\
& B_{X}^{\lambda}=\left\{M \in L(R): \lim _{n} \frac{1}{\lambda_{n}}\left|\left\{k \in I_{n}: X_{k}>M\right\}\right| \neq 0\right\} \\
& \overline{B_{X}^{\lambda}}=\left\{M \in L(R): \lim _{n} \frac{1}{\lambda_{n}}\left|\left\{k \in I_{n}: X_{k}<M\right\}\right|=1\right\} .
\end{aligned}
$$

The sets $\overline{A_{X}^{\lambda}}$ and $\overline{B_{X}^{\lambda}}$ are the sets of $\lambda$-statistical lower bound and $\lambda$-statistical upper bounds, respectively.

Theorem 2.1. If the sequence $X=\left(X_{k}\right)$ is $\lambda$-statistically bounded, then $\inf A_{X}^{\lambda}=\sup \overline{A_{X}^{\lambda}}$ and $\sup B_{X}^{\lambda}=\inf \overline{B_{X}^{\lambda}}$.

Proof. We will prove the first equality. Let $m=\inf A_{X}^{\lambda}$ and $M=\sup \overline{A_{X}^{\lambda}}$. By definition of $A_{X}^{\lambda}$ and $\overline{A_{X}^{\lambda}}$ we have $m \leq \tilde{m}$ for $\forall \tilde{m} \in A_{X}^{\lambda}$ and $M \geq \tilde{M}$ for $\forall \tilde{M} \in \overline{A_{X}^{\lambda}}$.

For every $\tilde{m} \in A_{X}^{\lambda}$ and $\tilde{M} \in \overline{A_{X}^{\lambda}}$, we have

$$
\lim _{n} \frac{1}{\lambda_{n}}\left|\left\{k \in I_{n}: X_{k}<\tilde{m}\right\}\right| \neq 0 \text { and }
$$

$$
\lim _{n} \frac{1}{\lambda_{n}}\left|\left\{k \in I_{n}: X_{k}>\tilde{M}\right\}\right|=1 \text {. This means that }
$$

$\delta_{\lambda}\left(\left\{k \in I_{n}: X_{k}<\tilde{m}\right\} \cap\left\{k \in I_{n}: X_{k}>\tilde{M}\right\}\right) \neq 0$. In other words, there is a number $k \in I_{n}$ such that $\tilde{M}<X_{k}<\tilde{m}$. Therefore, 


$$
\tilde{M}<\tilde{m} \text { for all } \tilde{m} \in A_{X}^{\lambda}, \tilde{M} \in \overline{A_{X}^{\lambda}}
$$

From (2.1), it follows that $\tilde{M}$ is a lower bound of the set $A_{X}^{\lambda}$.Then by definition of infimum we have $\tilde{M} \leq m=\inf A_{X}^{\lambda}$ This inequality holds for all $\tilde{M} \in \overline{A_{X}^{\lambda}}$. Then by definition of supremum we have

$$
M \leq m
$$

Now we show that the case $M<m$ cannot be place.

To the contrary, assume that $M<m$. This means that there is a number $\alpha \in[0,1]$ such that $\underline{M^{\alpha}}<\underline{m^{\alpha}}$ or $\overline{M^{\alpha}}<\overline{m^{\alpha}}$. For

The sake of definiteness we will consider the case

$$
\underline{M^{\alpha}}<\underline{m^{\alpha}}
$$

and then we will show that this leads to a contradiction. Denote $b=m\left(M^{\alpha}\right)$. It is clear that $b<\alpha$ ( $b$ can be zero). Moreover, for all $\lambda \in(b, \alpha]$ the inequality $\underline{M^{\lambda}}<\underline{m^{\lambda}}$ holds. Since the functions $M(x)$ and $m(x)$ are upper semi-continuous then there exists a point $(z, \beta)$ such that $z \in\left(M^{\alpha}, \underline{m^{\alpha}}\right), \beta \in(b, \alpha)$ and

$$
\underline{M^{\lambda}}<z, \underline{m^{\lambda}}>z \text { for all } \lambda \in[\beta, \alpha]
$$

Let us define the fuzzy numbers $\gamma_{1}$ and $\gamma_{2}$ as

$$
\gamma_{1}(x)=\left\{\begin{array}{ll}
0, & x<\underline{X^{0}} \\
\beta, & x \in\left[\underline{X^{0}}, z\right) \\
1, & x=z \\
0, & x>z
\end{array} \quad \gamma_{2}(x)= \begin{cases}0, & x<z \\
\beta, & x \in\left[z, \overline{X^{0}}\right) \\
1, & x=\overline{X^{0}} \\
0, & x>\overline{X^{0}}\end{cases}\right.
$$

where the numbers

$$
\underline{X^{0}}=s \lambda-\liminf \underline{X_{k}^{0}}-1
$$

$\overline{X^{0}}=s \lambda-\lim \sup \overline{X_{k}^{0}}+1$ arefinite. It is not difficult to observe that

$$
m \propto \gamma_{1}, m \propto \gamma_{2}
$$

This follows from

$$
\begin{gathered}
\underline{M^{\beta}} \geq s \lambda-\liminf \underline{X_{k}^{\beta}} \geq s \lambda-\lim \inf \underline{X_{k}^{0}}>\underline{X^{0}}=\underline{\gamma_{1}^{\beta}}, \\
\underline{M^{\alpha}}<z=\underline{\gamma_{1}^{\alpha}} \text { and } \underline{m^{b}} \leq \underline{M^{\alpha}}<z=\underline{\gamma_{2}^{b}}, \quad \underline{m^{\beta}}>z=\underline{\gamma_{2}^{\beta}} .
\end{gathered}
$$

Now let us consider the sets

$$
\begin{gathered}
C_{1}=\left\{k \in I_{n}: \underline{X_{k}^{\lambda}} \leq z, \text { forsome } \lambda \in(\beta, \alpha]\right\} \\
C_{2}=\left\{k \in I_{n}: \underline{X_{k}^{\lambda}}>z, \text { for some } \lambda \in(\beta, \alpha]\right\} .
\end{gathered}
$$

Clearly $C_{1} \cup C_{2}=I_{n}$ and therefore,

$$
\delta_{\lambda}\left(C_{1}\right)+\delta_{\lambda}\left(C_{2}\right) \geq 1
$$

First we assume that $\delta_{\lambda}\left(C_{1}\right)>0$. Taking in to account the structure of the fuzzy number $\gamma_{2}$ and the real number $\overline{X^{0}}$, we can obtain that $X_{k}<\gamma_{2}$ for all $\forall k \in C_{1} \backslash \mathrm{K}_{1}$, where $K_{1}=\left\{k \in I_{n}: \overline{X_{k}^{\lambda}}>\overline{X^{0}}\right.$, some $\left.\lambda \in[0,1]\right\}$. It is clear that $\delta_{\lambda}\left(K_{1}\right)=0$, hence we have $\delta_{\lambda}\left(C_{1} \backslash K_{1}\right)=\delta_{\lambda}\left(C_{1}\right)$. Thus, $\lim _{n} \frac{1}{\lambda_{n}}\left|\left\{k \in I_{n}: X_{k}<\gamma_{2}\right\}\right| \geq \delta_{\lambda}\left(C_{1}\right)>0$. This means that $\gamma_{2} \in A_{X}^{\lambda}$ and therefore, by definition of $\inf A_{X}^{\lambda}$ we obtain that $\gamma_{2} \geq m=\inf A_{X}^{\lambda}$. This contradicts (2.5) (that is $\left.m \sim \gamma_{2}\right)$.

Hence, we have shown that $\delta_{\lambda}\left(C_{1}\right)=0$.

Now from (2.6), it follows that $\delta_{\lambda}\left(C_{2}\right)=1$. Taking into account the structure of the fuzzy number $\gamma_{1}$ and the real number $\underline{X^{0}}$, we obtain that $X_{k}>\gamma_{1}$ for all $\forall k \in C_{2} \backslash\left(\mathrm{C}_{1} \cup \mathrm{K}_{2}\right)$,

where $K_{2}=\left\{k \in I_{n}: \underline{X_{k}^{\lambda}}<\underline{X^{0}}\right.$, some $\left.\lambda \in[0, \beta]\right\}$.

It is obvious that $\delta_{\lambda}\left(K_{2}\right)=0$, hence we conclude $\delta_{\lambda}\left(C_{2} \backslash\left(\mathrm{C}_{1} \cup \mathrm{K}_{2}\right)\right)=1$. This implies

$$
\lim _{n} \frac{1}{\lambda_{n}}\left|\left\{k \in I_{n}: X_{k}>\gamma_{1}\right\}\right| \geq \delta_{\lambda}\left(C_{2} \backslash\left(\mathrm{C}_{1} \cup \mathrm{K}_{2}\right)\right)=1,
$$

Which means that $\gamma_{1} \in \overline{A_{X}^{\lambda}}$. Thus $\gamma_{1} \leq M=\sup \overline{A_{X}^{\lambda}}$. This contradicts (2.5) (that is, $M \nsucc \gamma_{1}$ ).

This completes the proof.

Definition 2.1. If $X=\left(X_{k}\right)$ is a $\lambda$-statistically bounded sequence of fuzzy numbers, then the $\lambda$-statistical limit inferior of $X=\left(X_{k}\right)$ is given by $s \lambda-\liminf X=\inf A_{X}^{\lambda}$.

Also, the $\lambda$-statistical limit superior of $X=\left(X_{k}\right)$ is given by $s \lambda-\lim \sup X=\sup B_{X}^{\lambda}$.

By Theorem 2. 1. we get $s \lambda-\lim \inf X=\sup \overline{A_{X}^{\lambda}}$ and $s \lambda-\lim \sup X=\inf \overline{B_{X}^{\lambda}}$. A simple example will help to illustrate the concepts just defined.

Theorem 2.2. Let $X=\left(X_{k}\right)$ be a $\lambda$-statistically bounded sequence of fuzzy numbers. If $m=s \lambda-\lim \inf X$ then

$$
\lim _{n} \frac{1}{\lambda_{n}}\left|\left\{k \in I_{n}: X_{k}<m-\varepsilon_{1}\right\}\right|=0
$$

and

$$
\lim _{n} \frac{1}{\lambda_{n}}\left|\left\{k \in I_{n}: X_{k}<m+\varepsilon_{1}\right\} \bigcup\left\{k \in I_{n}: X_{k} \nsucc m+\varepsilon_{1}\right\}\right| \neq 0
$$

for every $\varepsilon>0$.

Proof. To the contrary, we asuume that there exists $\varepsilon>0$ such that $\lim _{n} \frac{1}{\lambda_{n}}\left|\left\{k \in I_{n}: X_{k}<m-\varepsilon_{1}\right\}\right| \neq 0$. This means 
that $m-\varepsilon_{1} \in A_{X}^{\lambda}$. Then we get $m \leq m-\varepsilon_{1}$ which is a contradiction.

Now let us show that inequality (2.8) holds. Assume that it is not true, that is, there exists $\varepsilon>0$ such that

$$
\left.\begin{array}{l}
\lim _{n} \frac{1}{\lambda_{n}}\left|\left\{k \in I_{n}: X_{k}<m+\varepsilon_{1}\right\}\right|=0 \\
\lim _{n} \frac{1}{\lambda_{n}}\left|\left\{k \in I_{n}: X_{k} \nsucc m+\varepsilon_{1}\right\}\right|=0
\end{array}\right\}
$$

For each $\forall k \in N$, only the following three cases are possible:

$$
X_{k}<m+\varepsilon_{1}, \quad X_{k} \geq m+\varepsilon_{1}, \quad X_{k} \chi_{m+\varepsilon_{1}} \text {. Then }
$$

$\left\{k \in I_{n}: X_{k}<m+\varepsilon_{1}\right\} \cup\left\{k \in I_{n}: X_{k} \geq m+\varepsilon_{1}\right\} \bigcup\left\{k \in I_{n}: X_{k} \nsucc m+\varepsilon_{1}\right\}=I_{n}$

Thus, from (2.9), we have

$\lim _{n} \frac{1}{\lambda_{n}}\left|\left\{k \in I_{n}: X_{k} \geq m+\varepsilon_{1}\right\}\right|=1$. This means that $m+\varepsilon_{1} \in \overline{A_{X}^{\lambda}}$.

Hence we can write $m+\varepsilon_{1} \leq \sup \overline{A_{X}^{\lambda}}=\inf A_{X}^{\lambda}=m$.

This is a contradiction. So we get,

$\lim _{n} \frac{1}{\lambda_{n}}\left|\left\{k \in I_{n}: X_{k}<m+\varepsilon_{1}\right\} \bigcup\left\{k \in I_{n}: X_{k} \nsucc m+\varepsilon_{1}\right\}\right| \neq 0$

Theorem 2.3. Let $X=\left(X_{k}\right)$ be a $\lambda$-statistically bounded sequence of fuzzy numbers. If $M=s \lambda-\lim \sup X$ then

$$
\left.\begin{array}{l}
\lim _{n} \frac{1}{\lambda_{n}}\left|\left\{k \in I_{n}: X_{k}>M+\varepsilon_{1}\right\}\right|=0 \\
\lim _{n} \frac{1}{\lambda_{n}}\left|\left\{k \in I_{n}: X_{k}>M-\varepsilon_{1}\right\} \cup\left\{k \in I_{n}: X_{k} \nsucc M-\varepsilon_{1}\right\}\right| \neq 0
\end{array}\right\}
$$

for every $\varepsilon>0$.

This can be proved similarly as Theorem 2.2. In addition, the converse of Theorem 2.3. does not hold in general. Now we have the following assertion.

Theorem 2.4. For any $\lambda$-statistically bounded sequence of fuzzy numbers $X=\left(X_{k}\right)$,

$$
s \lambda-\lim \inf X \leq s \lambda-\lim \sup X .
$$

Proof. We have $s \lambda-\lim \inf X=\sup \overline{A_{X}^{\lambda}} \quad$ and $s \lambda-\lim \sup X=\sup B_{X}^{\lambda}$. By definition of the sets $\overline{A_{X}^{\lambda}}$ and
$B_{X}^{\lambda}$, we see that $\overline{A_{X}^{\lambda}} \subseteq B_{X}^{\lambda}$. Hence we get $\sup \overline{A_{X}^{\lambda}} \leq \sup B_{X}^{\lambda}$ which completes the proof.

\section{ACKNOWLEDGMENT}

This work is supported by the Scientific Research Center at the Erciyes University with the project code FBA-12-4137.

\section{REFERENCES}

[1] H. Steinhaus, "Sur la convergence ordinarie et al. convergence asymptotique," Colloq. Math., vol. 2, 1951, pp. 73-74.

[2] H. Fast, "Sur la convergence statistique," Colloq. Math., vol. 2, 1951, pp. $241-244$.

[3] R. C. Buck, "Generalized asymptotic density," Amer. J. Math. vol. 75, 1953, pp. 335-346.

[4] M. Matloka, Sequences of fuzzy numbers, Busefal, vol. 28, 1986, pp. 28-37.

[5] S. Nanda, On sequence of fuzzy numbers, Fuzzy Sets and Systems, vol. 33, 1989, pp. 123-126.

[6] F. Nuray and E. Savaş, "Statistical convergence of fuzzy numbers," Math. Slovaca, vol. 45, no. 3, pp. 269-273, 1995.

[7] N. Tuncer and F. B. Benli, "A Note On $\lambda$-Statistically Cauchy Sequences," Inter. J. Pure and Appl. Math, vol. 31, no. 1, pp. 91-96, 2006.

[8] N. Tuncer and F. B. Benli, "Lambda-Statistical limit points of the sequences of fuzzy numbers," Information Sciences, vol. 177, pp. 3297-3304, 2007.

[9] F. B. Benli, Lambda-Statistically monotonic and Lambda statistically bounded sequences of fuzzy numbers, in press.

[10] J. X. Fang and H. Huang, "On the level convergence of a sequence of fuzzy numbers," Fuzzy Sets and Systems, vol. 147, pp. 417-435, 2004.

[11] $\mathrm{C}$. Wu, "The supremum and infimum of the set of fuzzy numbers and its application," J. Math. Anal. Appl. vol. 210, pp. 499-511, 1997.

[12] S. Aytar, M. A. Mamedov, and S. Pehlivan, "Statistical limit inferior and limit superior for sequences of fuzzy numbers," Fuzzy Sets and Systems, vol. 157, pp. 976-985, 2006

[13] J. A. Fridy and C. Orhan, "Statistical limit superior and limit inferior," in Proc. Amer. Math. Soc. vol. 125, pp. 3625-3631, 1997.

[14] M. L. Puri and D. A. Ralescu, "Differentials of fuzzy functions," $J$ Math. Anal. Appl., vol. 91, pp. 552-558, 1983.

[15] A. Kaufmann and M. M. Gupta, "Introduction to fuzzy arithmetic," Van Nostrand Reinhold, New York 1984.

[16] A. R. Freedman and J. J. Sember, "Densities and summability," Pacific J. Math., vol. 95, pp. 293-305, 1981.

[17] S. Aytar and S. Pehlivan, "Statistically monotonic and statistically bounded sequences of fuzzy numbers," Inf. Sci., vol. 176, pp. 734-744, 2006.

[18] E. Savaş, "On Strongly $\lambda$ - summable sequences of fuzzy numbers," Inf. Sci., vol. 125, pp. 181-186, 2000.

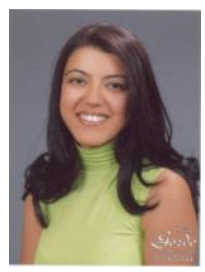

F. Berna Benli was born in Kayseri in 1978. She studied Mathematics at the Erciyes University (1995-1999), and have MSci, which is about Analysis and Functional Theory ("The equivalance of summability methods", Erciyes University, Turkey, 1999-2001).

Her Ph.D. thesis is about the fuzzy numbers ("Lambda Statistical Conergence of sequences of Fuzzy Numbers, Erciyes University, Turkey, 2006-2010).

She worked from 2001 until 2007 as Research Assistant at the Erciyes University and got the Assis. Prof. Dr. title in 2007. Since this date there she is working as Assis. Prof. Dr. at the Erciyes University in Kayseri (Turkey). Her research field are about the summability, fuzzy numbers and statistical convergence. 